\title{
Falsificationism Redux Indeed: a Rebuttal of the Callahan Rejoinder
}

\section{J. C. Lester}

Readers of "Falsificationism Redux" (the rejoinder) may have found it to be another waffling nonexplanation of induction and the alleged falsity of falsificationism - or even self-refuting, as its title indicates (redux: brought back, revived, restored). However, it seems worth another round of replies if only because the arguments are fairly typical of the would-be 'inductivist' and it might help some people who have yet to see how these arguments fail.

The rejoinder states that "denying any validity to induction clears the way to a full-blown skepticism about the claim that science yields any genuine knowledge about the world we occupy". But just because all knowledge, even a priori knowledge, remains ultimately conjectural and unsupported, does not mean that we need to doubt that we possess any true theories about the world. ${ }^{2}$ On the contrary, it is the inductivist's demand for his incoherent and impossible epistemology that "clears the way to a full-blown skepticism". Why would one "join with most scientists and many philosophers in regarding reliance on induction as an essential aspect of how science moves closer and closer to the truth"? No one would do that once they grasp that induction makes no sense. All alleged induction is fundamentally "naïve". There is no logical difference between the simplest and the most complicated versions. How can there be such things as "supportive instances of a theory"? How can even "risky predictions" support? What is "inductive reasoning"? The rejoinder still gives us no actual explanation of what induction is and how it works.

In what way, exactly, is Karl Popper "demonstrating his ignorance" by stating that the "fundamental doctrine which underlies all theories of induction is the doctrine of the primacy of repetitions"? Where would 'induction' be without repetition? It would have only conjecture and testing. I.e., it would be falsificationism. That self-styled 'inductivists' also use falsification does not make falsification part of "sophisticated inductivism" nor does it make repetition not basic to inductivism. It is quite a piece of cheek (or confusion) to claim falsification as part of "sophisticated induction" that trumps the "primacy of repetition" (although this confusion goes back at least to Francis Bacon [15611626], with his "induction by elimination"). Yes, "any verdict reached on the basis of the evidence available is fallible ... But that is no reason to conclude that juries never arrive at the correct verdict". This is hardly a point against falsificationism. Of course, "jurors...consider...also whatever positive (confirming) evidence for the defendant's guilt or innocence was presented" (although a falsificationist would explain this in more conjectural terms). But, although there are some analogies, this is not the epistemological theory of science that the rejoinder is supposed to be discussing. How can we support a universal scientific theory?

We are informed that "it is quite reasonable for a scientist to increase her confidence in her theory when she finds her experiments yield the results called for by it". Her "confidence" is irrelevant. How can such finite positive instances support an infinite universal theory? As a matter of logic, they cannot. But this lady scientist, we are told, is not like a crude inductivist, "reasoning from the bare fact that every swan she has seen was white to the generalization that all swans are so". Rather, "she believes, for instance, that she has discovered a genetic feature of swans that will always bring about white feathers, and, quite sensibly, she regards encountering thousands of swans, all of which are white, as evidence that she may be on to something". This example perfectly demonstrates the nonsense that there is a real difference between "crude" and "sophisticated induction". Consider: 1. All observed swans are white; therefore, all swans are (or are probably, or are more likely to be) white. 2. All observed swans have a genetic feature that makes them white; therefore, all swans have (or probably have, or are more likely to have) a genetic feature that makes them white. There is no logical difference between these two arguments. The rejoinder goes on to invite us to "notice" that "in the latter case, discovering a black swan need not even be taken to falsify her theory ... she only needs to add the proviso ... 'unless

\footnotetext{
1 Callahan, Gene. 2005. "Falsificationism Redux", Libertarian Alliance website: https://www.laarticles.org.uk/falsificationism_redux.htm.

${ }^{2}$ And, as Popper observed, that a theory is false does not mean it cannot have verisimilitude. This idea has proven logically problematic, but it makes intuitive sense in way that a description of something can be overall false (or inaccurate) but have some elements of truth (or degrees of accuracy).
} 
the swan has wildly abnormal genes"'. It is simply absurd to suggest that one can avoid falsification of a particular theory by changing it to a different theory. (In any case, there appears to be no theory that Australian black swans have "wildly abnormal genes"; so that would not save her theory unless it is implicitly true by definition, and so not scientific at all.) Moreover, one could also do the same silly trick with the first theory: one "only needs to add the proviso ... "unless the swan [is] wildly abnormal"".

"Boyle's conception of science" doesn't help to explain induction. It is even more perverse to try to apply it to a "rape trial" when we are supposed to be discussing universal scientific theories. Popper's generalised epistemology of critical rationalism has applications outside of science, but that is not the topic under discussion. It can only confuse matters to muddle the two. What of the remark that "those facts certainly do not constitute logical proof of the defendant's guilt, which is the standard Popper sets for inductive inference"? No, it is not "logical proof" that is the standard "for inductive inference", but merely making logical sense. Logically, how can a universal theory be supported by finite evidence?

It is no news that induction is a fallacy. It is no news that we cannot make any sense of it. The fact that some people still hanker after some form of induction is neither here nor there philosophically. To think it significant that many of them are prominent philosophers is the fallacy of arguing from authority (although to call all of those on the rejoinder's list 'inductivists' would be mistaken). So is citing philosophers who once apparently rejected induction but then later accepted it again. To repeat a point in the reply, Popper's "situational logic" (here being used to mean the logical implications of our being finite observers in an infinite world) did not itself "demolish inductivism". Rather, it allowed him to explain what we have instead of induction: hypothetico-deductivism and testing. And it is indeed confused to think that 1. explaining that induction does not make sense, and 2. explaining what we have instead of induction, are the same thing. When Popper claimed to have "solved ... the problem of induction" he meant just 2 . There is no problem with calling 2 "a breakthrough on the topic of induction" as long as this is not misunderstood as conflating 1 and 2.

What is the point of "arguing that inductive inference, when employed sensibly, with caution and humility, has a valid role in the evaluation of scientific theories" when we are simply not told what "inductive inference" is and how it could be other than illogical? One may as well replace "inductive inference" with "extrasensory perception"-except that ESP is not inherently illogical. The rejoinder goes on to discuss 'corroboration' (the word is etymologically unfortunate, as it means to add strength or support; far better simply to distinguish between tested theories and untested theories). It states that for the falsificationist "the best corroborated theory ... was the only rational means available for making predictions regarding future events falling within the scope of that current champion". It is, at most, sometimes imprudent to use an untested or a falsified theory (but Newton's falsified theory is often still applied as sufficiently approximating the truth). The 'corroborated' (test-passing) theory has no greater epistemological support than any unfalsified theory. Even if we do use the tested theory to make a prediction, that is not to assume it is known to be reliable. It is merely to use it. And we are not in any sense trying to "increase our belief" in a theory by testing it. One's beliefs are a matter of personal psychology, not science.

How is it an analogy with a scientific theory, 'corroborated' or otherwise, to state that "chess is a rational pastime, but that doesn't imply that it is rational to use chess results to forecast the weather"? There is no theoretical connection between "chess results" and the weather. And the same applies, mutatis mutandis, with the rejoinder's 'analogy' about "Aristotle's system of logic". In response to Popper's view that we should use scientific theories for our predictions, the rejoinder agrees with Wesley Salmon that "we could decide to read tea leaves or examine the inner organs of chickens instead". But the theory that these have any connection with making predictions has been refuted, albeit mostly in an informal way perhaps. Those who really claimed to predict by using them have been seen to be charlatans or deluded. Hence it is 'irrational' (better, imprudent) to use them. And the same argument applies with the suggestion that unless we have "inductive inference" then "it is no less rational to consult an astrologer or palm reader than an engineer when designing a new bridge" (although this is clearly about technology and not science).

A more relevant general question could be to ask why we might prefer tested to untested theories in the realm of practical applications. And the simple answer is that with tested theories we have at least falsified the theory that they are universally dangerous. But we have not done so with an untested theory. However, that is no guarantee that the tested theory really is safer. And testing itself 
never ends. In the realm of science, however, (which is what we are supposed to be discussing) we need not fear a theoretical error, because only the theory is at risk of being rejected - and even that is revisable. On the contrary, we want to seek out bold new theories in order to stand a chance of capturing interesting new truths. Therefore, we ought to behave quite differently than with immediate practical matters.

What of the charge that "falsification itself depends on inductive inferences"? In explanation of this we are told that our measurement could be in error, and we cannot "consider previous tests of the reliability of the measuring device" since this "clearly relies on inductive inference in extrapolating from the past performance of a device to the present case". (Strictly, induction is not about "extrapolating from the past... to the present" or the future, but about the logic of attempting to use known cases to infer support for unknown cases.) The key error here is in implicitly requiring that falsifications be justified. Falsifications are themselves conjectures open to testing, but we cannot test all our conjectures at once. If someone doubts that some test is accurate, then he is welcome to try to test the test. It is not relevant to falsificationism that this might be difficult with measurement, as the rejoinder also claims, but that is a problem for those people who want their theories supported. Unless the test can be faulted, we are logically entitled to conjecture that it was indeed a falsification.

The rejoinder itself correctly states that "there is no possibility of testing all of our ideas at the same time". But it incorrectly infers from this that tests "always rely on the past success of other theories as a valid indication of the confidence we can place in them". We do not "rely" on other theories when doing our tests. We merely use those theories. We are not trying to justify our falsification. It is also an error to think that this means that we have a "choice as to which theory, among the multitude relevant to an experiment, a negative result should be taken as falsifying". This is because, ex hypothesi, we are only testing one theory. And that theory is then - as a matter of logic-falsified (or not) by assuming the truth of the background theories. We can go on to test any background theory we like, of course. But that is where the choice lies, and not in changing the assumptions of the original test. It is hardly "arbitrary" to test the theory we were intending to test instead of switching to 'testing' another assumption instead (which is not only arbitrary but incoherent: the whole idea of a test vanishes).

In an infinite universe, that our universal scientific theories are probably false is not a sufficient reason to reject them. As conjecture is all we have to go on, we need evidence against a theory in order to rule it out. Therefore, to "suspect that there is an error in the particular auxiliary hypothesis" is, in the falsificationist sense, to suspect that it is empirically detectable. And, in any case, far from all auxiliary hypotheses will themselves be universal theories.

Yes, the falsificationist does not "count on the past as offering some basis for judging what he is now observing" and readily admits he "has no reason to suspect he might not be mistaken". This is because observations are not justified or supported. They too are conjectures (and 'universal' in some sense: they have unlimited implications, including counterfactual ones). Therefore, they are in court until we can find falsifying evidence. It might still be asked why some single observation, which is also fallible, ought to be regarded as falsifying the universal theory rather than the universal theory falsifying it. The answer is that we can never perceive all instantiations of a universal theory (e.g., see all swans being white), but we can and, ex hypothesi, do appear to see a single falsifying counterinstance (a black swan) and one which withstands testing. Although logically allowed, it is scientifically perverse simply to assume the truth of an unobservable universal theory in order to falsify apparently unproblematic observations. That is to engage in metaphysical dogmatism instead of empirical science.

Contra Kant and the rejoinder, it is not true that science as such "pre-supposes the existence of regular causal forces in the universe". Science is about trying to find out what there is in the universe. Randomness in most of it cannot be ruled out a priori. But even if there were "regular causal forces in the universe" without exception, that would have no relevance to anything the rejoinder has to say. We could still only detect them by conjecture and refutation. And a falsificationist can agree that "the fact that we often make mistakes does not imply that we never get it right".

On Copernicus, the rejoinder holds it to be a "peripheral error" when the reply to the original article states of the stars that, "it would have been arbitrary to give priority to the first conjecture as to their distance just because it was the first". The rejoinder asserts it was not arbitrary. It states that, according to the then standard theory, "the very essence of space made the existence of a vacuum impossible. Therefore, the sphere of the fixed stars had to be immediately beyond the far edge of the sphere of Saturn". It is not clear why being farther away entails a vacuum. The aether that was supposed 
to fill the space between Earth and the other planets could, presumably, fill the space between Saturn and the stars. In any case, that heliocentrism clashed with Aristotle's "whole system" is not a problem for falsificationism. There is nothing wrong with contradicting incompatible theories that are "well corroborated". Being "well corroborated" adds no strength to a theory. However, there were known to be severe problems with the Aristotelian system. Copernicus was, in fact, offering a bold alternative in an entirely falsificationist way. That the new theory was not yet independently testable was unfortunate. Often independent testability comes with time; but it might not come at all if we require it immediately for any theory even to be entertained. Popper stressed the importance of falsifiability in empirical science; he did not anathematise theoretical physics where independent testing is not yet possible.

The rejoinder objects to this last point. It says the "whole point of this criterion was ... to sort out truly scientific theories from pseudo-scientific ones, such as Marxism and Freudianism". So, either the latter two theories are scientific after all or "we are forced back into the strange position of excluding Copernicanism from the realm of science until the 1800s". First, it was only the distance to the stars that was untestable (which was also a problem shared with the Ptolemaic theory); not every aspect of Copernicanism was untestable, therefore it was empirically scientific. However, the rejoinder also misses the real point here. Marxism and Freudianism, although aiming for truth, somehow aspired to complete unfalsifiability. It seems unlikely that they had unfalsifiability as an explicit aim; they were just muddled. A true theory ought not to be falsified and ought to explain all relevant phenomena. Hence it might have superficially appeared that all was well. That said, as various people have since argued, they failed to be unfalsifiable and so they are really empirically scientific theories after all; and falsified ones too. But Copernicus and modern theoretical physicists want to find empirical tests for their theories. That they thus aspire, and often eventually succeed, makes it reasonable to say they are engaged in purely theoretical science rather than experimental science. The Popperian criterion of falsifiability does still mark a crucial distinction: between what is empirical science and what isn't.

As none of the rejoinder's arguments is sound, its conclusion that summarises them is similarly unsound. Hence, no further comment on that summary is needed except for one point added to it. The rejoinder claims that "in the end, even Popper agreed that his system contained 'a whiff of induction"'. No reference is given. The nearest that Popper used something like this expression in print appears to be just once: in the Library of Living Philosophers series edited by Paul Schillp, p.1192, note 165b. In fact, Popper put 'whiff' in inverted commas and referred to "inductivism" rather than "induction". At worst, this is a small slip on Popper's part. It is quoted in Miller 1994 with an explanation of why Popper was wrong to admit to even a "whiff". ${ }^{3}$ And a cursory internet search would have refuted the fanciful theory that the current author is the "only remaining Popperian who has not folded on this issue!"

Finally, the rejoinder begs the question when it states that a warning about a high crime rate "only reflects past crimes". It would reflect only past crimes if past crimes were all that were intended. Rather, what is implied by the warning is an ongoing propensity to crime in the area; which is clearly a conjecture going beyond the evidence. How is that conjecture "an inductive inference"? The rejoinder cannot explain. It has no theory of induction. There is no "inductive inference from past incidence of crime in the area to the future incidence" (nor even "from known incidence of crime in the area to the unknown incidence'). The warning is implicitly conjecturing ongoing causal factors that are responsible for crime. As that conjecture logically cannot be based on "the past prevalence of crime", then it is not based on it. The rejoinder is confusing evidence that is merely suggestive of a theory (it is a simple theory that anyone might first leap to in order to explain the phenomena), with evidence that somehow positively ('inductively') supports a theory. The rejoinder should have paid more attention to the logic of the arguments and tried harder to explain clearly and precisely what 'inductivism' is supposed to be and how it is possible.

(August 2005; revised October 2021)

\footnotetext{
${ }^{3}$ Miller, David. 1994. Critical Rationalism: A Restatement and Defence, Open Court, (p.24 and p.47). And for more explanations of critical rationalism see also Miller, David. 2005. Out of Error: Further Essays on Critical Rationalism, Ashgate.

${ }^{4}$ In fact, the current author is not really a "Popperian" (Popper had many philosophical theories), although he is a falsificationist and, more broadly, a critical rationalist.
} 